\title{
Introduction to the Special Issue: Myocardial Imaging in Heart Failure
}

\author{
Mouaz H. Al-Mallah ${ }^{1,2,3,4}$
}

Published online: 15 June 2017

(C) Springer Science+Business Media, LLC 2017

In the past decade, there have been major advancements in the field of cardiac imaging. New imaging techniques were developed and new tracers have become ready for routine clinical use. These new tools and techniques have and will continue to impact the current management of patients with heart failure. All of the above allowed clinicians to better understand the pathophysiology of heart failure, detect earlier alterations in cardiac structure and function, and identify prognostic markers in patients with suspected or confirmed heart failure [1]. Thus, it is no wonder that the current heart failure guidelines have multiple class I indications for the use of these new techniques across the spectrum of the diagnosis and management of heart failure patients [2, 3].

Given these changes, Heart Failure Reviews have dedicated this issue to update the readership on the role of these imaging tests (primarily cardiovascular magnetic resonance imaging (CMR) and positron emission tomography (PET)) in the current management of heart failure patients. We put together ten articles describing how PET and CMR can serve heart failure patients.

Aljizeeri and colleagues provided a primer about the role of CMR in patients with ischemic and non-ischemic

Mouaz H. Al-Mallah

mouaz74@gmail.com

1 King Abdulaziz Cardiac Center, King Abdulaziz Medical City, Ministry of National Guard-Health Affairs, Department Mail Code: 1413, P.O. Box 22490, Riyadh 11426, Kingdom of Saudi Arabia

2 Heart and Vascular Institute, Henry Ford Hospital, Detroit, MI, USA

3 King Saud bin Abdulaziz University for Health Sciences, Riyadh, Kingdom of Saudi Arabia

4 King Abdullah International Medical Research Center, Riyadh, Kingdom of Saudi Arabia cardiomyopathy [4]. This introductory article focuses on the distinction between different types of cardiomyopathy using the myocardial late gadolinium enhancement technique (LGE). LGE has been around for nearly two decades and remains the backbone of CMR imaging of cardiomyopathy [5]. While its presence is not specific for one cardiomyopathy, its location and pattern helps the imager determine the cardiomyopathy etiology and often guide therapy in these patients [6-8]. In addition, LGE is the main sequence used in CMR viability imaging. The utility of viability imaging have been recently questioned especially after the results of the PARR-2 trial [9](F-18-fluorodeoxyglucose positron emission tomography imaging-assisted management of patients with severe left ventricular dysfunction and suspected coronary disease) and the Surgical Treatment for Ischemic Heart Failure (STICH) trial [10]. However, viability remains a routinely ordered test in patients with ischemic cardiomyopathy who are being considered for revascularization. Jamiel and colleagues [11] reviewed the current controversy around viability imaging in the current era and identified knowledge gaps in the viability literature. They provide recommendations that could help when applying the test results in everyday clinical care of patients.

However, many patients with cardiomyopathy do not have LGE. This is mostly seen among patients with heart failure with preserved ejection fraction. Thus, there is need for new tools to help diagnose and prognosticate these patients. These new sequences are often referred to as multiparametric mapping techniques which have rapidly evolved and made it to the clinical arena in the past few years. These include T1 mapping, T2 mapping, and T2* imaging. We discuss these imaging sequences in two papers in this issue. Radenkovic and colleagues [12] reviewed the different T1 mapping sequences that are in clinical practice. These techniques (native $\mathrm{T} 1$, post contrast $\mathrm{T} 1$, and extracellular volume estimation) 
have recently been adopted in numerous pathologies, as they provide non-invasive tissue characterization with the potential to replace the need for invasive biopsy. They are steadily becoming diagnostically and prognostically useful tests for different pathologies like amyloidosis and other storage disease $[13,14]$, myocarditis, sarcoidosis, heart failure with preserved left ventricular ejection fraction, and even ischemic heart disease.

Lota [15] and colleagues also reviewed the role of CMR edema imaging in patients with heart failure. Edema imaging can be used in various conditions ranging from acute inflammatory and ischemic cardiomyopathy, to cardiac involvement in systemic diseases such as sarcoidosis and myocarditis. The paper also focuses on $\mathrm{T} 2 *$ imaging which is the main sequence used in the assessment of patients with suspected cardiac iron overload and cardiac hemochromatosis. Of note, this sequence can be performed in nearly $10 \mathrm{~min}$ without the need for contrast. Since the adoption of T2* imaging in the UK, there has been a significant decrease in the heart failure mortality of patients with cardiac iron overload [16].

Similar advancements have been seen in cardiac PET [17]. It is a comprehensive test that allows for the assessment of myocardial perfusion, viability, and ischemia. When combined with computed tomography, it provides valuable data on plaque imaging and coronary anatomy and calcium scoring $[18,19]$. The prognostic value of these tests has been well documented [20-23]. However, the recent interest in PET is partly due to its ability to assess myocardial blood flow accurately and provide an assessment of vasomotor tone and coronary flow reserve (CFR). The ability to measure myocardial blood flow, both at rest and under hyperemic conditions, and CFR allows for systematic evaluation of the integrity of the entire coronary vascular bed, beyond the ordinary assessment of obstructive lesions in epicardial vessels. The value of PET derived CFR is discussed in two papers in this issue. $\mathrm{Al}$ Badarin and colleagues [24] reviewed the principals of the measurement of myocardial blood flow and coronary flow reserve. The paper also reviews the role of CFR in ischemic heart disease and ischemic cardiomyopathy with focus on the new prognostic value data that emerged in the past few years. In another paper, Bravo and colleagues [25] reviewed the role of CFR and myocardial blood flow in non-ischemic cardiomyopathy. This is an area of intense research activity trying to define the diagnostic and prognostic value of CFR in cardiac amyloidosis [26], sarcoidosis, and other infiltrate diseases. Most of these patients have reduced blood flow at rest and peak hyperemia, more than what is seen in patients with ischemic heart disease. Data is starting to emerge on the diagnostic and prognostic role of CFR in this patient population.

In addition, the issue attempts a glimpse about the future of PET and CMR imaging in heart failure. There have been some tools that are not yet ready for prime time but are under investigation and development. In the past few months, there has been a new post-processing tool that allows for accurate assessment of myocardial strain using cine CMR imaging. This software can calculate the global longitudinal strain similar to what is done by echocardiography. There are a lot of on-going research activities in this area and are nicely summarized in the article by Scatteia and colleagues [27].

In addition, multiple vendors have been manufacturing hybrid PET MRI systems. These expensive machines combine the strength of both imaging modalities but their role in cardiac imaging is not very clear. Quail and colleagues [28] provide a review of the systems (their pros and cons) and discuss what could be their potential role in cardiac pathology including ischemic heart disease and inflammatory cardiomyopathies.

Similar to the new hardware, there have been multiple new tracers that are actively tested. Saraste and colleagues [29] reviewed the role of some of the new PET tracers in the assessment of underlying pathophysiology and therapeutic options in heart failure. The review focused on multiple tracers including the long awaited $18 \mathrm{~F}-$-Flurpiridaz and its role in assessment of ischemic heart disease. The authors also discuss sympathetic innervation imaging and its role to determine the risk of cardiac death, arrhythmia, and disease progression. Similarly, new tracers assess angiogenesis and other mechanisms involved in myocardial repair after infarction. In addition, there are many new tracers under development for the imaging of inflammatory cardiomyopathies. Qureshi and colleagues [30] also reviewed the role of CMR spectroscopy in the assessment of myocardial metabolism. While these protocols have been mostly used in research, recent developments resulted in shorter scan protocols. When combined with higher field strengths, it provides an opportunity to utilize this technology in studying and characterizing the metabolic derangements in heart failure patients.

Thus, this issue provides the reader a summary of the advances in the fields of CMR and PET and their role in the diagnosis and management of heart failure patients. Many of these tools are ready for clinical use and are widely adopted in different centers across the world. We hope that you enjoy reading this special issue and be able to apply some of these new tools in your clinical heart failure practice.

\section{Compliance with ethical standards}

Conflict of interest The author declares that he has no conflict of interest.

\section{References}

1. Dowsley T, Al-Mallah M, Ananthasubramaniam K, Dwivedi G, McArdle B, Chow BJ (2013) The role of noninvasive imaging in coronary artery disease detection, prognosis, and clinical decision making. Can J Cardiol 29:285-296 
2. Al-Mallah MH, Aljizeeri A, Villines TC, Srichai MB, Alsaileek A (2015) Cardiac computed tomography in current cardiology guidelines. J Cardiovasc Comput Tomogr 9:514-523

3. von Knobelsdorff-Brenkenhoff F, Schulz-Menger J (2016) Role of cardiovascular magnetic resonance in the guidelines of the European Society of Cardiology. J Cardiovasc Magn Reson 18:6

4. Aljizeeri A, Sulaiman A, Alhulaimi N, Alsaileek A, Al-Mallah MH (2017) Cardiac magnetic resonance imaging in heart failure: where the alphabet begins! Heart Fail Rev:1-15. doi:10.1007/s10741017-9609-4

5. Al-Mallah MH, Shareef MN (2011) The role of cardiac magnetic resonance imaging in the assessment of non-ischemic cardiomyopathy. Heart Fail Rev 16:369-380

6. Kwong RY, Heydari B, Abbasi S, Steel K, Al-Mallah M, Wu H, Falk RH (2015) Characterization of cardiac amyloidosis by atrial late gadolinium enhancement using contrast-enhanced cardiac magnetic resonance imaging and correlation with left atrial conduit and contractile function. Am J Cardiol 116:622-629

7. Crawford T, Mueller G, Sarsam S, Prasitdumrong H, Chaiyen N, Gu X, Schuller J, Kron J, Nour K, Cheng A (2014) Magnetic resonance imaging for identifying patients with cardiac sarcoidosis and preserved or mildly reduced left ventricular function at risk of ventricular arrhythmias. Circ Arrhythm Electrophysiol 7(6):1109 15. doi:10.1161/CIRCEP. 113.000156

8. Al-Mallah M, Kwong RY (2008) Clinical application of cardiac CMR. Rev Cardiovasc Med 10:134-141

9. Beanlands RS, Nichol G, Huszti E, Humen D, Racine N, Freeman M, Gulenchyn KY, Garrard L, deKemp R, Guo A, Ruddy TD, Benard F, Lamy A, Iwanochko RM, Investigators P (2007) F-18fluorodeoxyglucose positron emission tomography imagingassisted management of patients with severe left ventricular dysfunction and suspected coronary disease: a randomized, controlled trial (PARR-2). J Am Coll Cardiol 50:2002-2012

10. Velazquez EJ, Lee KL, Deja MA, Jain A, Sopko G, Marchenko A, Ali IS, Pohost G, Gradinac S, Abraham WT, Yii M, Prabhakaran D, Szwed H, Ferrazzi P, Petrie MC, O'Connor CM, Panchavinnin P, She L, Bonow RO, Rankin GR, Jones RH, Rouleau J-L (2011) Coronary-artery bypass surgery in patients with left ventricular dysfunction. N Engl J Med 364:1607-1616

11. Jamiel A, Ebid M, Ahmed AM, Ahmed D, Al-Mallah MH (2017) The role of myocardial viability in contemporary cardiac practice. Heart Fail Rev. doi:10.1007/s10741-017-9626-3

12. Dina Radenkovic B, Sebastian Weingärtner P, Ricketts L, Moon JC, Captur G (2017) HREV-D-17-00049R1 T1 Mapping in Cardiac MRI. Heart Fail Rev. doi:10.1007/s10741-017-9627-2

13. Fontana M, Chung R, Hawkins PN, Moon JC (2015) Cardiovascular magnetic resonance for amyloidosis. Heart Fail Rev 20:133-144

14. Zhao L, Fang Q (2016) Recent advances in the noninvasive strategies of cardiac amyloidosis. Heart Fail Rev 21:703-721

15. Lota AS, Gatehouse PD, Mohiaddin RH (2017) T2 mapping and T2* imaging in heart failure. Heart Fail Rev:1-10. doi:10.1007/ s10741-017-9616-5

16. Modell B, Khan M, Darlison M, Westwood MA, Ingram D, Pennell DJ (2008) Improved survival of thalassaemia major in the UK and relation to $\mathrm{T} 2 *$ cardiovascular magnetic resonance. J Cardiovasc Magn Reson 10:42

17. Al-Mallah MH, Sitek A, Moore SC, Di Carli M, Dorbala S (2010) Assessment of myocardial perfusion and function with PET and PET/CT. J Nucl Cardiol 17:498-513. doi:10.1007/s10741-0179621-8
18. Al-Mallah MH, Aljizeeri A, Alharthi M, Alsaileek A (2014) Routine low-radiation-dose coronary computed tomography angiography. Eur Heart J Suppl 16:B12-B16

19. Qureshi W, Blaha MJ, Nasir K, Al-Mallah MH (2013) Gender differences in coronary plaque composition and burden detected in symptomatic patients referred for coronary computed tomographic angiography. Int J Cardiovasc Imaging 29:463-469

20. Al-Mallah MH, Hachamovitch R, Dorbala S, Di Carli MF (2009) Incremental prognostic value of myocardial perfusion imaging in patients referred to stress single-photon emission computed tomography with renal dysfunction clinical perspective. Circ Cardiovasc Imaging 2:429-436

21. Al-Mallah MH, Qureshi W, Pantelic M, Nour K (2014) Long term prognostic value of coronary computed tomography angiography in suspected coronary artery disease: a 62 month median follow-up study. Int J Cardiol 176:1244-1246

22. Al-Mallah MH, Qureshi W, Lin FY, Achenbach S, Berman DS, Budoff MJ, Callister TQ, Chang HJ, Cademartiri F, Chinnaiyan K, Chow BJ, Cheng VY, Delago A, Gomez M, Hadamitzky M, Hausleiter J, Kaufmann PA, Leipsic J, Maffei E, Raff G, Shaw LJ, Villines TC, Cury RC, Feuchtner G, Plank F, Kim YJ, Dunning AM, Min JK (2014) Does coronary CT angiography improve risk stratification over coronary calcium scoring in symptomatic patients with suspected coronary artery disease? Results from the prospective multicenter international CONFIRM registry. Eur Heart J Cardiovasc Imaging 15:267-274

23. Cheruvu C, Precious B, Naoum C, Blanke P, Ahmadi A, Soon J, Arepalli C, Gransar H, Achenbach S, Berman DS, Budoff MJ, Callister TQ, Al-Mallah MH, Cademartiri F, Chinnaiyan K, Rubinshtein R, Marquez H, DeLago A, Villines TC, Hadamitzky M, Hausleiter J, Shaw LJ, Kaufmann PA, Cury RC, Feuchtner G, Kim YJ, Maffei E, Raff G, Pontone G, Andreini D, Chang HJ, Min JK, Leipsic J (2016) Long term prognostic utility of coronary CT angiography in patients with no modifiable coronary artery disease risk factors: results from the 5 year follow-up of the CONFIRM International Multicenter Registry. J Cardiovasc Comput Tomogr $10: 22-27$

24. Al Badarin F, Aljizeeri A, Almasoudi F, Al-Mallah MH (2017) Assessment of myocardial blood flow and coronary flow reserve with positron emission tomography inischemic heart disease: current state and future directions. Heart Fail Rev. doi:10.1007/ s10741-017-9625-4

25. Bravo PE, Di Carli MF, Dorbala S (2017) Role of PET to evaluate coronary microvascular dysfunction in non-ischemic cardiomyopathies. Heart Fail Rev:1-10

26. Noordzij W, Glaudemans AWJM, Longhi S, Slart RHJA, Lorenzini M, Hazenberg BPC, Rapezzi C (2015) Nuclear imaging for cardiac amyloidosis. Heart Fail Rev 20:145-154

27. Alessandra Scatteia M, Baritussio A, Bucciarelli-Ducci C (2017) Strain imaging using cardiac magnetic resonance HREV-D-1700053. Heart Fail Rev. doi:10.1007/s10741-017-9621-8

28. Quail MA, Sinusas AJ (2017) PET-CMR in heart failure - synergistic or redundant imaging? Heart Fail Rev:1-13. doi:10.1007/ s10741-017-9607-6

29. Saraste A, Knuuti J (2017) PET imaging in heart failure: the role of new tracers. Heart Fail Rev:1-11. doi:10.1007/s10741-017-9620-9

30. Qureshi WT, Ub N (2017) Principals and clinical applications of magnetic resonance cardiac spectroscopy in heart failure. Heart Fail Rev, 9:1. doi:10.1007/s10741-017-9611-x 